\title{
RESEARCH
}

Open Access

\section{Prognosis of limited-stage small cell lung cancer with comprehensive treatment including radical resection}

Lili Zhong ${ }^{1,2+}$, Jiaojiao Suo ${ }^{1,2+}$, Ya Wang ${ }^{1,2+}$, Jialong Han ${ }^{1,2}$, Huijie Zhou ${ }^{1,2}$, Hao Wei ${ }^{1,2}$ and Jiang Zhu ${ }^{1 *}$ (D)

\begin{abstract}
Background: The NCCN (National Comprehensive Cancer Network) Clinical Practice Guidelines in Oncology (NCCN guidelines) recommend radical resection for T1-2NOMO patients with limited-stage small cell lung cancer (LS-SCLC). However, only about $5 \%$ of patients with small cell cancer (SCLC) were initially diagnosed as T1-2NOMO. The purpose of our study was to analyze and compare the effects of the comprehensive treatment including radical surgery and concurrent chemoradiotherapy on the prognosis of patients with LS-SCLC.
\end{abstract}

Methods: We comprehensively reviewed the medical data of patients with SCLC diagnosed by pathology in our hospital from January 2011 to April 2018. The Ethics Committee of West China Hospital of Sichuan University approved the study. Finally, 50 patients with good follow-up and complete medical data were selected as the surgical group (S group). According to the clinical characteristics of the patients in the S group, 102 LS-SCLC patients who received concurrent chemoradiotherapy in the same period were included in the CCRT group (concurrent chemoradiotherapy group) as the control group. Then according to the orders of the adjuvant treatments, the patients in the $S$ group were divided into the SA group (radical surgery + adjuvant chemotherapy + adjuvant radiotherapy group, 30 cases in total) and the NS group (neoadjuvant chemotherapy + radical surgery + adjuvant chemotherapy \pm adjuvant radiotherapy group, 20 cases in total) for subgroup analysis. The SPSS 23.0 software was used for statistical analysis, and the $t$ test was used for group comparison; Kaplan-Meier was used for survival analysis. $P<0.05$ demonstrates a statistically significant difference.

Results: The median progress-free survival (PFS) in the $S$ group (73 months) was significantly better than that in the CCRT group (10.5 months, $P<0.0001$ ), and the median overall survival (OS) in the $S$ group (79 months) was also significantly better than that in the CCRT group (23 months, $P<0.0001$ ). Subgroup analysis showed that there was no significant difference between the NS group and the SA group.

Conclusions: For LS-SCLC patients, the comprehensive treatment including radical surgery (radical surgery + adjuvant chemotherapy \pm adjuvant radiotherapy/neoadjuvant chemotherapy + radical surgery + adjuvant chemotherapy \pm adjuvant radiotherapy)may be superior to concurrent chemoradiotherapy.

Keywords: Limited-stage small cell lung cancer, Radical surgery, Neoadjuvant chemotherapy, Concurrent chemoradiotherapy, Prognosis

\footnotetext{
* Correspondence: zhujiang@wchscu.cn

†Lili Zhong, Jiaojiao Suo and Ya Wang contributed equally to this work.

'Department of Thoracic Oncology, West China Hospital, Sichuan University,

Chengdu, China

Full list of author information is available at the end of the article
}

(c) The Author(s). 2020 Open Access This article is distributed under the terms of the Creative Commons Attribution 4.0 International License (http://creativecommons.org/licenses/by/4.0/), which permits unrestricted use, distribution, and reproduction in any medium, provided you give appropriate credit to the original author(s) and the source, provide a link to the Creative Commons license, and indicate if changes were made. The Creative Commons Public Domain Dedication waiver (http://creativecommons.org/publicdomain/zero/1.0/) applies to the data made available in this article, unless otherwise stated. 


\section{Introduction}

Lung cancer is the most common cause of cancer and cancer deaths among men worldwide and the second most common cause of cancer death in women worldwide [1, 2]. The pathology of lung cancer is mainly divided into small cell lung cancer (SCLC) and non-small cell lung cancer (NSCLC). SCLC accounts for about $15 \%$ of lung cancer patients [2]. Most (about 70\%) patients with SCLC are diagnosed as having extensive small cell lung cancer (ESSCLC). Only about 30\% of SCLC patients are diagnosed as having limited-stage small cell lung cancer (LS-SCLC), but their prognosis is still not optimistic with a median survival time of 15-20 months [3]. Since the 1970s, platinum-based combination chemotherapy with etoposide (EP) or irinotecan (IP) has been established as the main treatment of SCLC. For decades, platinum combined with etoposide chemotherapy and combined with concurrent radiotherapy has been the standard treatment for LS-SCLC $[4,5]$. Although SCLC is sensitive to radiotherapy and chemotherapy, it is prone to drug resistance. The effect of later line treatment is not optimistic, and the survival time of patients is short [6]. Due to the failure of early clinical studies of surgery in LS-SCLC patients, the NCCN (National Comprehensive Cancer Network) Clinical Practice Guidelines in Oncology (NCCN guidelines) recommend only the patients with LS-SCLC who have staged T1-2N0M0 cancer to undergo radical surgery. However, in the initial diagnosis of SCLC patients, less than 5\% meet this criterion [7]. The standard treatment recommended by the current NCCN guidelines for LS-SCLC is concurrent chemoradiotherapy [8], and prophylactic cranial irradiation (PCI) is considered for LS-SCLC patients who have good responses to induction therapy. Although the effective rate of first-line treatment is as high as 80\%, most patients would relapse within 6 months after the completion of the initial treatment [9]. Therefore, a better treatment strategy is still our unremitting goal of exploration. This study was intended to analyze and compare the effects of the comprehensive treatment including radical surgery and concurrent chemoradiotherapy on the prognosis of patients with LS-SCLC.

\section{Methods}

\section{Patients}

The Ethics Committee of West China Hospital of Sichuan University approved the study.

From January 2011 to April 2018, patients with SCLC were chosen based on information from the Department of Medical Records West China Hospital of Sichuan University. Then patients with LS-SCLC were selected by consulting medical records. Finally, 152 patients with good follow-ups and complete medical data were included in our study. The primary endpoints were progression-free survival (PFS) and overall survival (OS).

\section{Inclusion criteria}

1. Patients were biopsied by operation/fiber optic/ bronchoscope or by endobronchial ultrasoundguided transbronchial needle aspiration (EBUSTBNA)/mediastinoscopy or pulmonary puncture under image guidance and then diagnosed with SCLC after pathological investigation or pathological consultation at the West China Hospital of Sichuan University.

2. After PET-CT, contrast-enhanced thoracic and abdominal CT, head MRI, bone scan, and other imaging examinations, the patient was diagnosed with LS-SCLC.

3. Patient received anti-tumor treatment for SCLC at the West China Hospital, including surgery and/or chemotherapy and/or radiotherapy.

4. Patient medical records and follow-up data were completed.

\section{Exclusion criteria}

1. Patient received only palliative and symptomatic support treatment and did not receive any treatment or gave up treatment during the course.

2. Patient had severe cardio-cerebrovascular disease or other diseases that may have had a significant impact on prognosis.

3. Patient was lost to follow-up or patient medical records/important information was incomplete or missing.

4. Baseline examination revealed a possible (unidentified) presence of lesions in the patient beyond the limitation period.

5. Patient received targeted therapy.

\section{Grouping}

1. S group (surgical group), 50 patients in total:

a) SA group (radical surgery + adjuvant chemotherapy \pm adjuvant radiotherapy group), 30 cases in total 
b) NS group (neoadjuvant chemotherapy + radical surgery + adjuvant chemotherapy \pm adjuvant radiotherapy group), 20 cases in total

2. CCRT group (concurrent chemoradiotherapy group), 102 patients in total

\section{Therapeutic schedule}

\section{$S$ group (surgical group)}

1. SA group (radical surgery + adjuvant chemotherapy \pm adjuvant radiotherapy group)

(a) Each patient was admitted to the hospital to complete the relevant examination, and the radical operation (lobectomy combined with regional lymph node dissection) was performed after surgical contraindications were excluded. Then adjuvant therapy was arranged according to the results of the postoperative pathological examination.

(b) Adjuvant chemotherapy: EP regimen (etoposide + cisplatin, etoposide $100 \mathrm{mg} / \mathrm{m}^{2} \mathrm{~d} 1-\mathrm{d} 3$ + cisplatin $75 \mathrm{mg} / \mathrm{m}^{2} \mathrm{~d} 1-\mathrm{d} 3$, repeated every 21 days) or EC regimen (etoposide + carboplatin, etoposide $100 \mathrm{mg} / \mathrm{m}^{2} \mathrm{~d} 1-\mathrm{d} 3+$ carboplatin AUC $=4-6 \mathrm{~d} 1$, repeated every 21 days), with a maximum of four cycles.

(c) Adjuvant radiotherapy: Patients with lymph node metastasis confirmed by postoperative pathology were treated with adjuvant radiotherapy (mediastinal radiation therapy, 45 Gy, 1.5 Gy bid, 30 fractions for 3 weeks).

2. NS group (neoadjuvant chemotherapy + radical surgery + adjuvant chemotherapy \pm adjuvant radiotherapy group)

(a) Neoadjuvant chemotherapy (EP or EC regimen same as before) was given to patients after discussion by a multi-disciplinary treatment (MDT) consisting of the Department of Thoracic Surgery and Department of Thoracic Oncology with a maximum of four cycles.

(b) Thoracic and abdominal contrast-enhanced CTs were reexamined to evaluate the efficacy. After the MDT discussion, the patients who could be treated surgically were selected for radical surgery (lobectomy with regional lymph node dissection). (c) The Department of Thoracic Oncology then decided if each patient needed adjuvant therapy or not based on postoperative pathology.

\section{CCRT group (concurrent chemoradiotherapy group)}

Each patient received concurrent chemoradiotherapy (completing radiotherapy within 42 days after the start of chemotherapy). The chemotherapy regimen was EP or EC (same as before). The method was CF (conventional fraction) radiotherapy, with a dose of 60-70 Gy/ 30-35 times, once a day, 5 days/week.

\section{Follow-up}

West China Hospital's hospital information system (HIS), telephone, letter, and public security systems were used to collect patient data. Each patient's basic information was retrieved by the medical records department, and the patient's medical records and information were reviewed in the HIS system after screening according to the inclusion criteria and the exclusion criteria. Then each patient's follow-up information was obtained through medical records retrieval, telephone, and inquiry to the public security household registration system.

Follow-up deadline: September 2019.

\section{Statistical analysis}

This study is a single-center retrospective clinical study based on the real world. The SPSS 23.0 software was used for statistical analysis, and the $t$ test was used for group comparison; Kaplan-Meier was used for survival analysis. $P<0.05$ demonstrates a statistically significant difference.

\section{Results}

Basic information on the 152 patients with LS-SCLC

This study selected patients with SCLC who were diagnosed and treated in West China Hospital of Sichuan University from January 2011 to April 2018. The information was retrieved from the Information Department of West China Hospital of Sichuan University, removing repeated cases (including revisiting and repeated treatment patients). After reviewing each patient's medical records and imaging data through the HIS, we selected the patients who were diagnosed as LS-SCLC at the time of initial. Each patient's treatment and survival status were followed up by telephone, medical record system, and public security system. Patients who had unfinished treatment or only accepted supportive treatment or whose medical records were incomplete or missing important information or lost follow-up were excluded. Finally, 152 patients were enrolled in the study. The deadline for follow-up was September 2019. 
Thirty patients (19.7\%) underwent surgical treatment firstly and then underwent adjuvant therapy or not according to postoperative pathology; 20 patients (13.2\%) underwent neoadjuvant chemotherapy firstly and then received surgery. A total of 102 patients (67.1\%) did not undergo surgery and received concurrent chemoradiotherapy according to the standard treatment regimen.

Survival analysis was performed on all these $152 \mathrm{pa}-$ tients, and the median PFS was 18 months and the median OS was 30 months. The 1-year survival rate was $78.9 \%(120 / 152)$, the 2 -year survival rate was $58.6 \%(89 / 152)$, and the 5 -year survival rate was $27.6 \%$ $(42 / 152)$.

The screening process for patients in this study is shown in Fig. 1; basic information on the 152 patients with LS-SCLC is shown in Table 1.

\section{Survival}

Progression-free survival (PFS)

The relationship between the PFS curve and the treatment modality is shown in Fig. 2a, b.

\section{Overall survival (OS)}

The relationship between the OS curve and the treatment modality is shown in Fig. 3a, b.

\section{Discussion}

Lung cancer was very rare before the twentieth century, but due to environmental changes and genetic susceptibility the incidence and mortality has jumped to top among all cancers in the world $[10,11]$. In 2012, there were 1.8 million new lung cancer cases and 1.6 million lung cancer deaths worldwide accounting for $19 \%$ of all cancer deaths [12]. Lung cancer is divided into SCLC

Collecting medical information of SCLC patients who were

diagnosed in our hospital from January 2011 to April 2018

Removing repeated cases

(including re-visiting and repeated treatment patients)

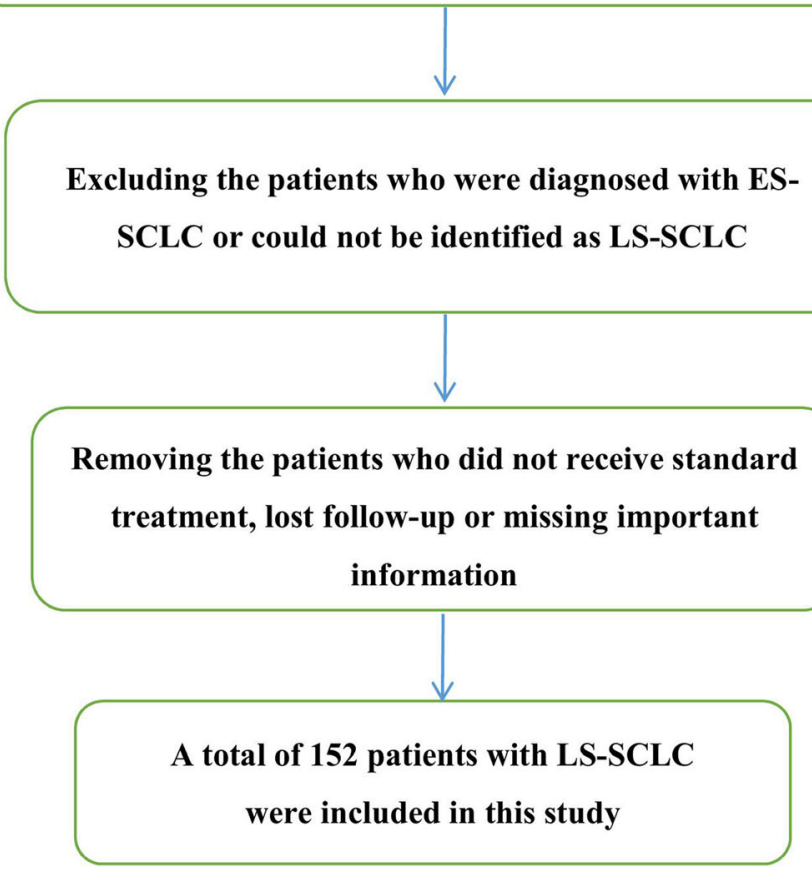

Fig. 1 The screening process for patients in this study 
Table 1 Basic information on the 152 patients with LS-SCLC

\begin{tabular}{lllll}
\hline Item & Classification & S group & CCRT group & $P$ \\
\hline Total number of cases & & 50 & 102 & \\
Age (years old) & $\geq 60<60$ & 1436 & 4557 & 0.097 \\
Gender & Male & 41 & 74 & 0.232 \\
& Female & 9 & 28 & \\
Primary tumor site & Left lung & 24 & 54 & 0.607 \\
& Right lung & 26 & 48 & \\
Basic diseases & No & 40 & 77 & 0.550 \\
(hypertension/diabetes) & Yes & 10 & 25 & \\
TNM staging & I & 25 & 50 & 0.937 \\
& II & 9 & 19 & \\
Smoking history & III & 16 & 33 & \\
& No & 31 & 48 & 0.088 \\
PCl & Yes & 19 & 54 & \\
& No & 12 & 35 & 0.262 \\
Chemotherapy cycles & $\geq 4$ & 38 & 67 & \\
& $<4$ & 37 & 91 & 0.069 \\
\hline
\end{tabular}

$S$ group surgical group, CCRT group, concurrent chemoradiotherapy group

and NSCLC. SCLC develops rapidly and has strong invasiveness and poor prognosis. About 30\% of SCLC patients are diagnosed with LS-SCLC at the time of diagnosis and the median OS is $15-20$ months. The remaining 70\% SCLC patients are diagnosed as ESSCLC at the time of diagnosis and the median OS is only 8-13 months [12-14]. Because most LS-SCLC patients have regional lymph node metastasis at the time of diagnosis, radical surgery has been considered a surgical contraindication while chemotherapy is the main treatment. There are some studies that have overturned the status of surgery in patients with this disease [15, 16]; however, these studies did not screen for TNM staging in the LS-SCLC patients and the patients only underwent radical surgery without any adjuvant. But some previous studies showed that some patients with early-stage SCLC may benefit from surgery $[17,18]$. At present, the NCCN guidelines recommend only the patients with LS-SCLC who have staged T1-2N0M0 to undergo radical surgery and concurrent chemoradiotherapy is the standard treatment model for other LS-SCLC patients [19]. Although SCLC is sensitive to chemotherapy, it is prone to drug resistance which leads to treatment failure and poor disease outcome [20]. In this article, we analyzed and compared the effects of the comprehensive treatment including radical surgery and concurrent chemoradiotherapy on the prognosis of patients with LS-SCLC. The results indicated that the surgery plays an important role in the treatment of patients with LS-SCLC and that comprehensive treatment involving surgery can significantly improve the prognosis of patients compared with concurrent chemoradiotherapy.

Compared with adjuvant chemotherapy combined with surgery, some previous studies have shown that neoadjuvant chemotherapy combined with surgery may could give the LS-SCLC patients a better chance of survival [21-23]. This result may be attributed to the effect of Stage $\mathrm{T}$ or $\mathrm{N}$ down-staging by neoadjuvant chemotherapy, which helps patients diminish several symptoms and obtain more survival benefit from radical resection [21]. But the controversy about neoadjuvant chemotherapy for LS-SCLC still exists. There also have studies which showed that compared with adjuvant chemotherapy combined with surgery neoadjuvant chemotherapy combined with surgery has no better survival [24]. Though our study demonstrates that there is no statistically significant difference between the two subgroups a trend shows that neoadjuvant chemotherapy combined with surgery may have the better chance of survival than adjuvant chemotherapy combined with surgery.

\section{S group VS CCRT group}

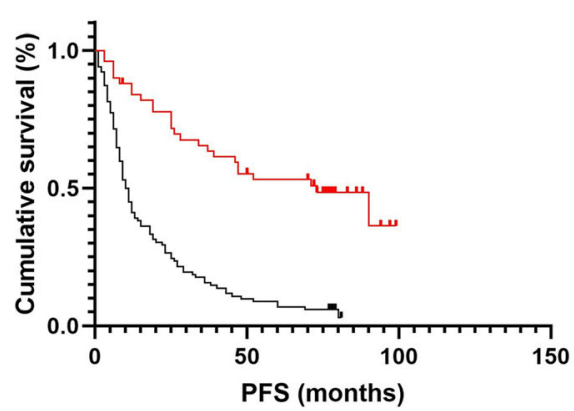

SA group VS NS group

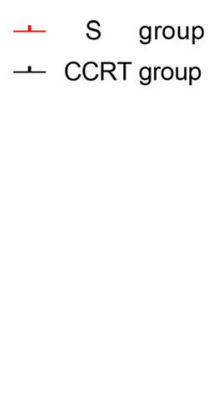

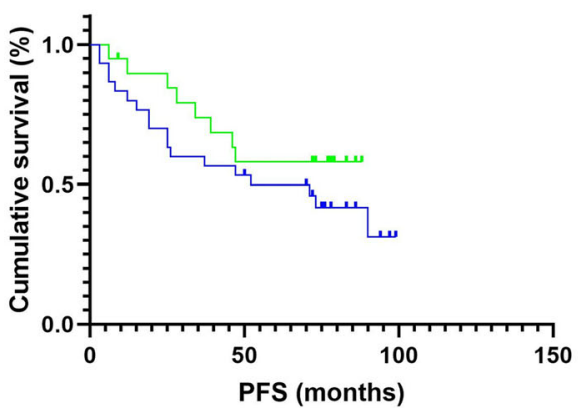

+ SA group

+ NS group

Fig. 2 a S group vs CCRT group. S group, surgical group; CCRT group, concurrent chemoradiotherapy group. The median PFS of the S group was 73 months, and the median PFS of the CCRT group was 10.5 months. $P<0.0001$ demonstrates a statistical difference. $\mathbf{b}$ SA group vs NS group. SA group, radical surgery + adjuvant chemotherapy \pm adjuvant radiotherapy group; NS group, neoadjuvant chemotherapy + radical surgery + adjuvant chemotherapy \pm adjuvant radiotherapy group. The median PFS of the SA group was 52 months, and the median PFS of the NS group was not reached. $P=0.252$ demonstrates no statistical difference 

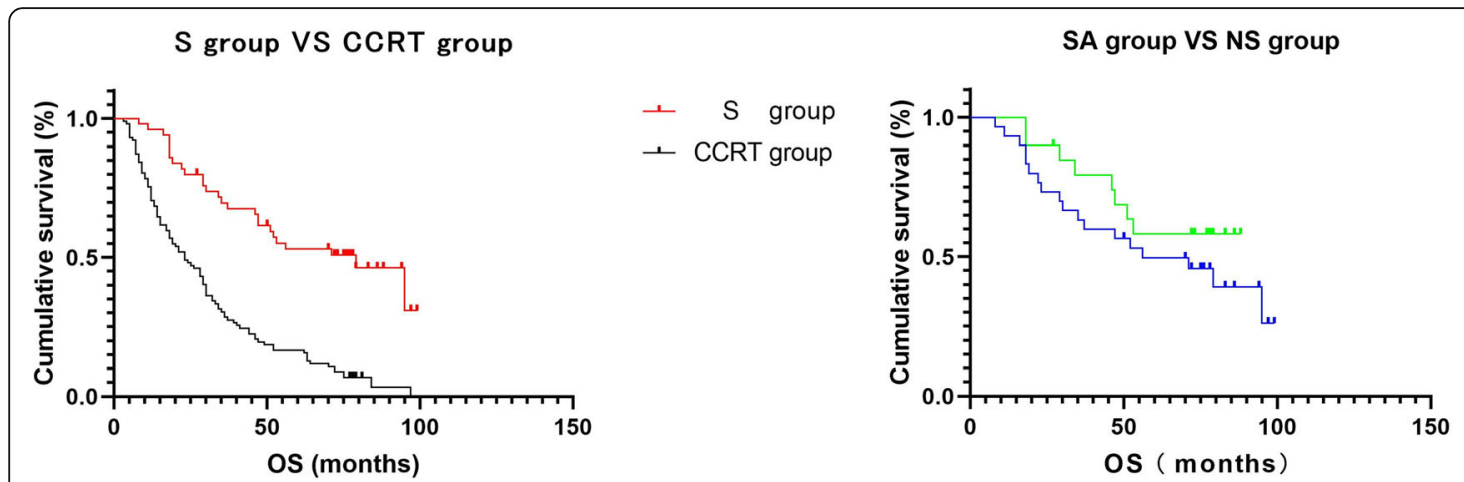

+ SA group

- NS group

Fig. 3 a S group VS CCRT group. S group, surgical group; CCRT group, concurrent chemoradiotherapy group. The median OS of the S group waS 79 months, and the median OS of the CCRT group was 23 months. $P<0.0001$ demonstrates a statistical difference. In the $S$ group, the 1-year survival rate was $96.0 \%$, the 2 -year survival rate was $80.0 \%$, and the 5 -year survival rate was $28.0 \%$. In the CCRT group, the 1-year survival rate was $70.6 \%$, the 2-year survival rate was $48.0 \%$, and the 5-year survival rate was $16.7 \%$. b SA group VS NS group. SA group, radical surgery + adjuvant chemotherapy \pm adjuvant radiotherapy group; NS group, neoadjuvant chemotherapy + radical surgery + adjuvant chemotherapy \pm adjuvant radiotherapy group. The median OS of the SA group was 56 months, and the median OS of the NS group was not reached. $P=0.266$

demonstrates no statistical difference

Because there were a relatively few number of patients in this study and it is a retrospective study, the general situations of the patients involved were not that balanced. There are also other inevitable defects such as the mixed bias caused by the heterogeneity of the social and economic conditions of the group, as well as evitable selection bias. All of that may have affected the effectiveness of this study. The efficacy of the comprehensive treatment including radical surgical for LS-SCLC patients needs to be confirmed with a larger sample size and multi-center research results.

\section{Conclusions}

For LS-SCLC patients, the comprehensive treatment including radical surgery (radical surgery + adjuvant chemotherapy \pm adjuvant radiotherapy/neoadjuvant chemotherapy + radical surgery + adjuvant chemotherapy \pm adjuvant radiotherapy) may be superior to concurrent chemoradiotherapy.

\section{Acknowledgements}

We appreciated all authors for their efforts on the manuscript.

\section{Authors' contributions}

YW analyzed the data. JJS, JLH, HJZ, and HW contributed reagents/materials/ analysis tools. LLZ wrote the paper. All authors read and approved the final manuscript.

\section{Funding}

There is no applicable funding in this study.

\section{Availability of data and materials}

All data came from the Department of Medical Records, West China Hospital of Sichuan University.

\section{Ethics approval and consent to participate}

The Ethics Committee of West China Hospital of Sichuan University approved the study. This article does not contain any studies with human participants or animals performed by any of the authors.
Consent for publication

Not applicable.

\section{Competing interests}

The authors declare that they have no competing interests.

\section{Author details}

'Department of Thoracic Oncology, West China Hospital, Sichuan University, Chengdu, China. ${ }^{2}$ West China School of Medicine, Sichuan University, Chengdu, China.

Received: 9 October 2019 Accepted: 26 January 2020

Published online: 03 February 2020

\section{References}

1. Didkowska J, Wojciechowska U, Manczuk M, et al. Lung cancer epidemiology: contemporary and future challenges worldwide []]. Ann Transl Med. 2016;4(8):150.

2. Temraz S, Charafeddine M, Mukherii D, et al. Trends in lung cancer incidence in Lebanon by gender and histological type over the period 2005-2008 [J]. J Epidemiol Glob Health. 2017:7(3):161-7.

3. Rudin CM, Ismaila N, Hann CL, et al. Treatment of small-cell lung cancer: American Society of Clinical Oncology Endorsement of the American College of Chest Physicians guideline [J]. J Clin Oncol. 2015;33(34):4106-11.

4. Yao W. Analysis of small cell lung cancer pfs affected factors [D]. City: Kunming Medical University; 2013.

5. Zhang SL, Zhang HS, Cordoncardo C, et al. Selection of tumor antigens as targets for immune attack using immunohistochemistry. 2. Blood grouprelated antigens [J]. International Journal of Cancer. 1997;73(1):50-6.

6. Shi L. Clinical observation of combined chemotherapy of lobaplatin and irinotecan in the treatment of relapsed advanced or progress small cell lung cancer [D]. Zunyi Medical College: City; 2012.

7. Takei $\mathrm{H}$, Kondo $\mathrm{H}$, Miyaoka $\mathrm{E}$, et al. Surgery for small cell lung cancer: a retrospective analysis of 243 patients from Japanese Lung Cancer Registry in 2004[]]. Journal of Thoracic Oncology. 2014;9(8):1140-5.

8. Zhuoqun Wang. Analysis of prognostic factors in 92 patients with small cell lung [D]. City: Dalian Medical University, 2017.

9. Zarogoulidis K, Ziogas E, Papagiannis A, et al. Interferon alpha-2a and combined chemotherapy as first line treatment in SCLC patients: a randomised trial [J]. Lung Cancer. 1996;15(2):197-205.

10. Siegel R, Ma J, Zou Z, et al. Cancer statistics, 2014 [J]. CA Cancer J Clin. 2014; 64(1):9-29.

11. Small cell lung cancer (version 2.2018) [M]. National Comprehensive Cancer Network. 15 Jun 2018. 
12. Ferlay J, Soerjomataram I, Dikshit $\mathrm{R}$, et al. Cancer incidence and mortality worldwide: sources, methods and major patterns in GLOBOCAN 2012 [J]. International Journal of Cancer. 2015;136(5):E359-86.

13. Siegel RL, Miller KD, Jemal A. Cancer statistics, 2016 [J]. CA Cancer J Clin. 2016;66(1):7-30.

14. Kalemkerian G P, Schneider B J. Advances in small cell lung cancer [J]. Hematology-Oncology Clinics of North America, 2017, 31(1): 143-+.

15. Fox W, Scadding JG. Medical Research Council comparative trial of surgery and radiotherapy for primary treatment of small-celled or oat-celled carcinoma of bronchus. Ten-year follow-up [J]. Lancet. 1973;2(7820):63-5.

16. Miller AB, Fox W, Tall R. Five-year follow-up of the Medical Research Council comparative trial of surgery and radiotherapy for the primary treatment of small-celled or oat-celled carcinoma of the bronchus [J]. Lancet. 1969; 2(7619):501-5.

17. Iwata T, Nishiyama N, Nagano K, et al. Role of pulmonary resection in the diagnosis and treatment of limited-stage small cell lung cancer: revision of clinical diagnosis based on findings of resected specimen and its influence on survival. Gen Thorac Cardiovasc Surg. 2012;60:43-60.

18. Liu T, Chen Z, Dang J, Li G. The role of surgery in stage I to III small cell lung cancer: a systematic review and meta-analysis. PLoS One. 2018 Dec 31; 13(12):e0210001. doi: 10.1371/journal.pone.0210001. PMID: 30596754; PMCID: PMC6312204.

19. National Comprehensive Cancer Network (NCCN): Clinical practice guidelines in oncology: small cell lung cancer (version 1.2016).Washington: NCCN, 2016

20. Jackman DM, Johnson BE. Small-cell lung cancer [J]. Lancet. 2005;366(9494): 1385-96.

21. Xu YJ, Zheng H, Gao W, et al. Is neoadjuvant chemotherapy mandatory for limited-disease small-cell lung cancer? Interact Cardiovasc Thorac Surg. 2014;19:887-19:88doi: 10.1093/icvts/ivu262.

22. Hara N, Ohta M, Ichinose Y, Motohiro A, Kuda T, Asoh H, et al. Influence of surgical resection before and after chemotherapy on survival in small cell lung cancer. J Surg Oncol. 1991;47:53-47.

23. Zheng QF, Li S, Zhang L, et al. Retrospective study of surgical resection in the treatment of limited stage small cell lung cancer. Thorac Cancer. 2013:4 395-4:395

24. Dou X, Wang Z, Wang L, Lu W, Ma Y, Xu S. Analysis of efficacy of surgical treatment for a small cell lung cancer. Zhongguo Fei Ai Za Zhi. 2017 Feb 20; 20(2):88-92. Chinese. doi: 10.3779/j.issn.1009-3419.2017.02.03. PMID: 28228219; PMCID: PMC5972969.

\section{Publisher's Note}

Springer Nature remains neutral with regard to jurisdictional claims in published maps and institutional affiliations.

Ready to submit your research? Choose BMC and benefit from:

- fast, convenient online submission

- thorough peer review by experienced researchers in your field

- rapid publication on acceptance

- support for research data, including large and complex data types

- gold Open Access which fosters wider collaboration and increased citations

- maximum visibility for your research: over $100 \mathrm{M}$ website views per year

At $\mathrm{BMC}$, research is always in progress.

Learn more biomedcentral.com/submissions 